J. Austral. Math. Soc. 19 (Series B), (1976), 381-386.

\title{
ON THE VARIATIONAL DERIVATION OF EINSTEIN'S STRONG FIELD EQUATIONS
}

\author{
L. J. GREGORY AND A. H. KLOTZ
}

(Received 5 June 1974)

(Revised 1 July 1976)

\begin{abstract}
It is shown that the necessary and sufficient condition for the transposition invariance of the field equations derivable from an Einstein-Kaufman variational action principle is the vanishing of the vector $\Gamma_{\lambda}$. When this condition is satisfied, the field equations become the so-called strong field equations of Einstein. In this sense, the latter can be claımed to follow from the same action principle.
\end{abstract}

\section{Introduction}

The purpose of this note is to show that the strong field equations (ref. 1) of Einstein's non-symmetric unified field theory can be derived from a variational action principle under the condition of general transposition invariance.

The strong field equations are, in Einstein's notation,

$$
g_{\mu \nu, \lambda}=0, \quad \Gamma_{\lambda}=0, \quad R_{\mu \nu}=0,
$$

Greek indices going from one to four.

The problem of their compatibility has been discussed in detail by Mme Tonnelat (ref. 2) and by Hlavaty (ref. 3). However, their results are inconclusive. Hlavaty, for example, shows that given a particular solution of the system (1), further solutions can be constructed (loc. cit., Theorem IV, 8.2, p. 150 and others). Such solutions have been found (e.g., ref. 4) but only under severe symmetry restrictions which reduce the number of the equations to be solved but lack the generality of the variational methods. Mme Tonnelat remarks outright (p. 303 of her book) that the equations (1) cannot be derived by variational methods. 
At first sight the field described by the above equations appears to be over-determined since there are eighty four equations $(64+4+16)$ for only eighty unknowns, namely sixty four $\Gamma_{\mu \nu}^{\lambda}$ 's which can be found by algebraic means (as Tonnelat has shown) and the sixteen components $g_{\mu \nu}$ of the fundamental tensor. For the latter, the equations

$$
R_{\mu \nu}=0 \text {, }
$$

that is the vanishing of the non-symmetric Ricci tensor, form a set of partial differential equations.

The equations (1) exhibit a well-known symmetry (transposition invariance, $v$. section 3) but it is uncertain a priori whether this reduces their number suitably (i.e. by 4) to make them formally compatible. At first, Einstein announced (loc. cit.) discovery of four identities among his equations. Later, however (ref. 5), he withdrew his conclusion, apparently based on a mistake, and, together with Straus, modified the non-symmetric theory ("the weak field equations").

It is shown below that a further version of the unified field theory (ref. 6) can be formulated in such a way that the desired identities reappear in a self-evident manner. Also, a necessary and sufficient conditions that the new field equations should be transposition invariant is

$$
\Gamma_{\lambda}=0 \text {, }
$$

when they collapse into the set (1). Since the former equations are derived from a variational action principle, it follows that the strong field equations are also derived from it.

The mathematical calculations relevant to the present work are contained in the above-mentioned publication (ref. 6). We shall omit repeating them here, merely stating the relevant results.

We should like to express our gratitude to the Referee for pointing out serious deficiencies in an earlier version of this work written by one of us, and also to the late Professor C. Lanczos of the Dublin Institute for Advanced Studies and to our colleagues, Drs. P. W. Buchen and W. B. Fraser for helpful comments.

\section{The variational principle}

Let, as usual,

$$
\xi^{\mu \nu}=\sqrt{-g} g^{\mu \nu},
$$

where $g^{\mu \nu}$ is the tensorial inverse of the fundamental tensor: 


$$
g^{\mu \nu} g_{\lambda \nu}=\delta_{\lambda}^{\mu}=g^{\nu \mu} g_{\nu \lambda},
$$

and

$$
g=\operatorname{det}\left(g_{\mu \nu}\right)<0
$$

Let us adopt also the variational principle of Einstein and Kaufman (ref. 7):

$$
\delta \int\left(S^{\mu \nu} R_{\mu \nu} d \tau=0 .\right.
$$

The variation is to be carried out with respect to the parameters $\left(\mathcal{G}^{\mu \nu}\right.$ and $U_{\mu \nu}^{\lambda}$ the latter being related to the components $\Gamma_{\mu \nu}^{\lambda}$ of the non-symmetric affine connection by the equation

$$
\begin{aligned}
\Gamma_{\mu \nu}^{\lambda}= & U_{\mu \nu}^{\lambda}+\left(2 a_{1}+\frac{1}{3}\right) \delta_{\nu}^{\lambda} U_{\underline{\mu \nu}}^{\sigma}+\left(3 a_{1}+1\right) \delta_{\mu}^{\lambda} U_{\underline{\nu}}^{\sigma} \\
& +\left(3 a_{1}+2 a_{2}+1\right) \delta_{\mu}^{\lambda} U_{\nu} .
\end{aligned}
$$

As in Einstein's work, a line under a pair of indices denotes the symmetric part of the indexed quantity, a hook, its skew symmetric part, and

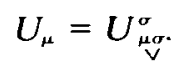

$a_{1}$ and $a_{2}$ are numerical parameters which play no part in the variation. The only restriction on these parameters is the condition of solvability of the equation (4), for $U_{\mu \nu}^{\lambda}$ in terms of $\Gamma_{\mu \nu}^{\lambda}$ :

$$
\left(15 a_{1}+4\right)\left(9 a_{1}+6 a_{2}+2\right) \neq 0 .
$$

The result of carrying out the variation (3) may be written in the form

$$
\int\left(R_{\mu \nu} \delta\left(\xi^{\mu \nu}+\mathcal{N}_{A}^{\mu \nu} \delta U_{\mu \nu}^{\Lambda}\right) d \tau=0\right.
$$

so that the field equations become

$$
R_{\mu \nu}=0 \quad \text { and } \quad \mathcal{N}_{\lambda}^{\mu \nu}=0 .
$$

It has been shown (ref. 6) that under the condition

$$
9 a_{1}+6 a_{2}+2 \neq 0
$$

which, of course, is automatically satisfied because of the condition (5), the second of the equations (7) implies the four identities

$$
\left(S_{v_{. \nu}}^{\mu \nu}=0\right. \text {. }
$$

Moreover, solving the equation (4) for $U_{\mu, n}^{\lambda}$ and substituting into 


$$
\mathcal{N}_{\lambda}^{\mu \nu}=0
$$

the latter becomes

$$
g_{\mu \nu: \lambda}-\frac{2}{3}\left(\Gamma_{\lambda} g_{\mu \nu}+\Gamma_{\nu} g_{\mu \lambda}\right)=0
$$

independently of $a_{1}$ and $a_{2}$. These are algebraic equations which should define the components $\Gamma_{\mu \nu}^{\lambda}$ of the affine connection in terms of the fundamental tensor $g_{\mu,}$ and its first derivatives $g_{\mu v, \lambda}$. In a separate note we show that they are of rank 60 (instead of 64) and fail to determine $\Gamma_{\lambda}$. In particular, they are not transposition invariant.

\section{The condition of transposition invariance}

The transposition conjugate of a covariant tensor

$$
A_{\mu \nu}=A_{\mu \nu}\left(g_{\alpha \beta}, \Gamma_{\alpha \beta}^{\gamma}\right)
$$

of rank 2, constructed from the fundamental tensor and the affine connection (and their derivatives) is defined (ref. 7) as

$$
A_{\mu \nu}^{\dagger}=A_{\nu \mu}\left(g_{\beta \alpha}, \Gamma_{\beta \alpha}^{\gamma}\right) \text {. }
$$

Clearly, any such tensor can be split invariantly into a sum of transposition symmetric (or invariant) and transposition anti-symmetric parts:

$$
A=\frac{1}{2}\left(A+A^{+}\right)+\frac{1}{2}\left(A-A^{\dagger}\right) .
$$

(In the original version (ref. 1) of the non-symmetric theory, Einstein employed complex $g_{\mu \nu}$, and $\Gamma_{\mu \nu}^{\lambda}$ and transposition invariance was equivalent to Hermitian symmetry of the equations.) In dealing with non-symmetric quantities, the condition of transposition invariance ensures that we should not get more equations than those obtained by separating their symmetric and anti-symmetric parts.

Let us now return to the equation (9). Its transposition conjugate is

$$
g_{\mu \nu, \lambda}+\frac{2}{3}\left(\Gamma_{\lambda} g_{\mu \nu}+\Gamma_{\mu} g_{\lambda \nu}\right)=0 \text {. }
$$

Hence, subtracting equation (9) from (13)

$$
2 g_{\mu \nu} \Gamma_{\lambda}+g_{\lambda \nu} \Gamma_{\mu}+g_{\mu \lambda} \Gamma_{\nu}=0 .
$$

Contracting equation (14) with $g^{\mu \nu,}$ we get

$$
8 \Gamma_{\lambda}+\delta_{\lambda}^{\mu} \Gamma_{\mu}+\delta_{\lambda}^{\nu} \Gamma_{t}=10 \Gamma_{\lambda}=0 .
$$

Thus 


$$
\Gamma_{\lambda}=0
$$

is the necessary and sufficient condition for the transposition invariance of the field equations with respect to $g_{\mu \nu}$ and $\Gamma_{\mu, \nu}^{\lambda_{\mu}}$ Under condition (2), the field equations become the strong field equations (1). The process is, of course, compatible with the condition (8) since the first of the equations (1) implies

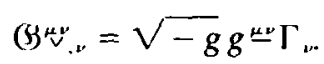

We have thus shown that, given the variational principle (3) on which the condition of Transposition Invariance with respect to $\left(S^{\mu \nu}\right.$ and $\Gamma_{\mu \nu}^{\lambda}$ is imposed, the only set of field laws is the strong field equations system (1).

\section{Concluding remarks}

It follows immediately from the relation (4) that

$$
\Gamma_{\mu}=-\frac{1}{2}\left(9 a_{1}+6 a_{2}+2\right) U_{\mu}+\frac{1}{2}\left(15 a_{1}+4\right) U_{\underline{\mu} \underline{\sigma}}^{\sigma} .
$$

The condition (2) therefore implies that

$$
U_{\underline{\underline{g}}}^{\sigma}=\frac{9 a_{1}+6 a_{2}+2}{15 a_{1}+4} U_{\mu}
$$

when the second set of the field equations (7) becomes (ref. 6)

$$
g_{\mu \nu, \lambda}(U)-\left(2 K g_{\mu,} U_{\lambda}+(K+1) g_{\mu \lambda} U_{\nu}+(K+1) g_{\lambda \nu} U_{\mu}\right)=0,
$$

where $g_{\mu \cdot \lambda}(U)$ denotes the usual expression with $U_{\mu \nu}^{\lambda}$, replacing $\Gamma_{\mu m}^{\lambda}$ and

$$
K=\frac{1}{3} \frac{\left(6 a_{1}+1\right)\left(9 a_{1}+6 a_{2}+2\right)}{\left(15 a_{1}+4\right)} .
$$

We can verify as in the last section that the condition of transposition invariance of the equation (17) but with respect to $U_{\mu \nu}^{\lambda}$ is

$$
K U_{\mu}=0 .
$$

Thus, we again obtain strong field equations except when

$$
a_{1}=-\frac{1}{6} \quad \text { and } \quad a_{2} \neq-\frac{1}{12}
$$

when $K=0$. As shown in (ref. 6), we can regard

$$
\left(\mathcal{G}^{\mu \nu} U_{\nu}\right.
$$


as the current density vector for this particular version of the theory though, of course,

$$
\left(3^{\mu \nu} \Gamma_{\nu}\right.
$$

suggested therein vanishes. The vector $U_{\mu}$ satisfies the identity

$$
g_{\mu \lambda} U_{\nu}-g_{\lambda \nu} U_{\mu}=0
$$

which does not entail vanishing of $U_{\mu}$.

\section{References}

[1] A. Einstein, Ann. Math. 46 (1945), 578.

[2] M. A. Tonnelat, Les Théories Unitaires de l'Électromagnetisme et de la Gratitation (Paris, 1965).

[3] V. Hlavaty, Geometry of Einstein's Unified Field Theory (Groningen, 1957).

[4] A. H. Klotz and G. K. Russell, Acta Phys. Polon. B3 (1972), 413.

[5] A. Einstein and E. G. Straus, Ann. Math. 47 (1946).

[6] A. H. Klotz and G. K. Russell, Acta Phys. Polon., B4 (1973), 579.

[7] A. Einstein and B. Kaufman, Ann. Math. 62 (1955) 128.

Department of Applied Mathematics,

University of Sydney,

Sydney,

Australia. 